\title{
Theory of Gossamer and resonating valence bond superconductivity
}

\author{
J. Y. Gan, ${ }^{1}$ F. C. Zhang, ${ }^{2,3}$ and Z. B. $\mathrm{Su}^{1}$ \\ ${ }^{1}$ Institute of Theoretical Physics, Chinese Academy of Sciences, Beijing, China \\ ${ }^{2}$ Department of Physics, The University of Hong Kong, Hong Kong, China \\ ${ }^{3}$ Department of Physics, University of Cincinnati, Cincinnati, Ohio 45221, USA
}

(Received 22 August 2003; revised manuscript received 9 August 2004; published 13 January 2005)

\begin{abstract}
We use an effective Hamiltonian for two-dimensional Hubbard model including an antiferromagnetic spinspin coupling term to study recently proposed Gossamer superconductivity. We apply a renormalized mean field theory to approximately take into account the strong correlation effect in partially projected Gutzwiller wave functions. At the half-filling, there is a first order phase transition to separate a Mott insulator at large Coulomb repulsion $U$ from a Gossamer superconductor at small $U$. At the critical value $U=U_{c}$, the charge carrier density and the superconducting (SC) order parameter change discontinuously from zero in the Mott insulating phase to finite values in the Gossamer SC phase. The first order transition is due to the interplay of the kinetic and spin exchange energies. As the electron density changes away from half-filling, the Gossamer SC state changes smoothly, while the Mott insulator is evolved into a resonating valence bond (RVB) SC state. The Gossamer and RVB SC states have the same pairing symmetry. The SC order parameter changes smoothly from a RVB SC state at $U>U_{c}$ to a Gossamer SC state at $U<U_{c}$ at a fixed nonhalf-filled electron density. We argue that the RVB SC state is smoothly connected to the Gossamer SC state, hence to the BCS state.
\end{abstract}

DOI: 10.1103/PhysRevB.71.014508

PACS number(s): 75.10.Jm, 05.30.Pr

\section{INTRODUCTION}

Since the discovery of high temperature superconductivity in the cuprates, ${ }^{1,2}$ there have been a lot of theoretical efforts to understand its microscopic mechanism. One of the scenarios was initiated by Anderson, ${ }^{3}$ who proposed the idea of a resonating valence bond (RVB) state for the observed unusual properties in these compounds. A minimum model for cuprates was proposed to be two-dimensional Hubbard or its equivalent $t-J$ model in the large $U$ limit. ${ }^{3,4}$ In the RVB picture, each lattice site is either unoccupied or singly occupied by a spin-up or spin-down electron. The spins are coupled antiferromagnetically without long range order. The charge carriers move in the spin background and condense to a superconducting (SC) state. ${ }^{5-12}$ In this scenario, the undoped cuprate with density of one electron per site is a Mott insulator, and the superconductor is viewed as a doped Mott insulator. Many experimentally observed properties in cuprates, such as the $d$-wave symmetry in superconductivity, ${ }^{13,14}$ the pseudogap phenomena, ${ }^{15}$ and the linear doping dependence of the superfluid density in the underdoped region, ${ }^{16}$ seem to be consistent with the RVB mean field theory, as discussed in a recent review. ${ }^{5}$ On the other hand, while mean field theories and variational calculations support the SC ground state in the doped Hubbard or $t-J$ models, more direct numerical calculations on these models remain controversial and have been unable to provide unambiguous answers to this question. ${ }^{17-22}$

Very recently, Laughlin has proposed an interesting notion, the Gossamer superconductivity, for high $T_{c} \mathrm{SC}$ $\mathrm{Cu}$-oxides. ${ }^{23}$ In a Gossamer superconductor, the superfluid density is tenuous, in contrast to the conventional superconductor. He proposed a partially Gutzwiller projected BCS wave function [Eq. (2) below] to describe the Gossamer SC state, and a Hamiltonian for which his wave function is an exact ground state. By expanding that Hamiltonian, Laughlin showed that the SC ground state requires a large attractive interaction in addition to a large on-site Coulomb repulsion.

In a previous short paper, ${ }^{24}$ one of us (F.C.Z.) argued that the effective Hamiltonian of the Hubbard model acting on the Gutzwiller's wave function should include a spin-spin coupling term, and that the on-site Coulomb repulsion generates an attractive pairing interaction in addition to the suppression of electron's onsite double occupation. Using Gutzwiller variational method, it was demonstrated that the ground state at half-filled electron density is a Gossamer superconductor for smaller intrasite Coulomb repulsion $U$ and a Mott insulator for larger $U$. The Gossamer SC state is similar to the RVB SC state. The transition between a Mott insulator and a superconductor at half-filled electron density has also been studied by Baskaran, ${ }^{25}$ who introduced a twospecies $t-J$ model and argued its superconductivity to be similar to the RVB. More recently, Bernevig et al. ${ }^{26}$ have examined the instability of the Gossamer superconductivity towards a magnetic insulator within the framework of Laughlin's Gossamer Hamiltonian. ${ }^{23}$

In this paper, we extend the idea explored in Ref. 24 to study the phase transition in strongly correlated electron systems in great detail. In particular, we use an effective Hubbard like Hamiltonian [Eq. (1) below] in a square lattice to systematically study the partially projected Gutzwiller wave function and the competition between the Mott insulator and the superconductor. We use Gutzwiller's approximation to replace the strong correlation in the projected state by a set of renormalized factors, and to carry out a renormalized mean field theory numerically to study the ground state and the elementary excited states of the system. Our main results can be summarized below. At the half-filling, the ground state is a Mott insulator at large $U$, and a Gossamer superconductor at small $U$. The transition is the first type in the physically interesting parameter region. The charge carrier density and the SC order parameter change discontinuously from zero in the Mott insulating phase to finite values in the 
SC state at the critical value of $U$. As the electron density changes away from half-filling, the Gossamer SC state changes smoothly, while the Mott insulating phase is evolved to the RVB SC state. The Gossamer and RVB SC states have the same pairing symmetry, and their SC order parameters are both suppressed by a unified renormalized factor, which quantitatively characterizes the smallness of the superfluid density. The SC order parameters in both RVB and Gossamer SC states are calculated and are found to change smoothly between the two states as $U$ is varied at a fixed non-halffilled electron density. We thus argue that the RVB SC state is smoothly connected to the Gossamer SC state. ${ }^{25}$ Since a Gossamer SC state is adiabatically connected to a BCS state $^{23}$ our theory suggests that the RVB SC state is smoothly connected to the BCS state. The Gossamer SC state at half-filling may be viewed as a RVB state with equal number of independent empty and doubly occupied sites. ${ }^{27}$ From this point of view, the reduction of $U / t$, which may be realized by applying the pressure, ${ }^{25}$ plays a similar role as the chemical doping. The Gossamer superconductivity may have already been realized in organic superconductors. ${ }^{25,28}$ In the present paper, we focus on the SC and insulating properties of the problem, and neglect the possible antiferromagnetism in the model.

This paper is organized as follows: In Sec. II, we introduce the model and the variational wave function. In Sec. III, we formulate a renormalized mean field theory to study the variational wave function. Section IV is devoted to the phase transition between the Mott insulator and the Gossamer superconductor at the half-filling. Detailed discussions on the Gossamer and RVB SC states are given in Sec. V. The paper is concluded with a summary in Sec. VI.

\section{THE MODEL AND THE VARIATIONAL WAVE FUNCTION}

We study an effective Hubbard Hamiltonian in a square lattice,

$$
\begin{gathered}
H=H_{t}+H_{s}+H_{U}, \\
H_{t}=-\sum_{\langle i j\rangle \sigma}\left(t_{i j} c_{i \sigma}^{\dagger} c_{j \sigma}+\text { h.c. }\right), \\
H_{s}=J \sum_{\langle i j\rangle} S_{i} \cdot S_{j}, \\
H_{U}=U \sum_{i} n_{i \uparrow} n_{i \downarrow} .
\end{gathered}
$$

In the above equations, $c_{i \sigma}$ is the annihilation operator of an electron of spin $\sigma$ at the lattice site $i$, and $n_{i \sigma}=c_{i \sigma}^{\dagger} c_{i \sigma}$. The sum is over the nearest neighbor pairs of $\langle i j\rangle$, and $U>0$ is the intrasite Coulomb repulsion. We assume $t>0$. The case at $t<0$ can be mapped onto the model with $t>0$. In this Hamiltonian, we have introduced an antiferromagnetic spinspin coupling term to account for the effect of the virtual electron hopping process. In the large $U$ limit, $J \approx 4 t^{2} / U$. This model may be viewed as an effective Hamiltonian of the Hubbard model. The inclusion of the antiferromagnetic spin coupling appears consistent with the weak coupling renormalization group analysis, ${ }^{29}$ and is appropriate in the variational approach studied here. In the limit $U \rightarrow \infty$, the model is reduced to the $t-J$ model. While there is a lack of derivation of the Hamiltonian (1) from the Hubbard model, we believe Eq. (1) to be relevant to the physics of the Hubbard model at large $U$ including values near the transition points we are most interested in. Equation (1) may be very different from the Hubbard model at small $U$, however. Keeping this in mind, below we shall consider Eq. (1) from a phenomenological point of view, and consider $J$ to be an independent parameter, and study its solutions within the framework of Gutzwiller's variational approach.

Due to the perfect nesting and the van Hove singularity in the density of state, the ground state of Hamiltonian (1) at half-filling (electron density $n=1$ per site) is an antiferromagnet for arbitrarily small value of $U$ in the absence of the spin-spin coupling term $(J=0)$. In this paper, however, we will not consider the magnetic long range order.

We study the model using a variational trial wave function proposed by Laughlin, ${ }^{23}$

$$
\begin{gathered}
\left|\Psi_{\mathrm{GS}}\right\rangle=\Pi_{\alpha}\left|\Psi_{\mathrm{BCS}}\right\rangle, \\
\Pi_{\alpha}=\prod_{i}\left(1-\alpha n_{i \uparrow} n_{i \downarrow}\right),
\end{gathered}
$$

with $\left|\Psi_{\mathrm{BCS}}\right\rangle$ a BCS SC state, given by

$$
\left|\Psi_{\mathrm{BCS}}\right\rangle=\prod_{\boldsymbol{k}}\left(u_{\boldsymbol{k}}+v_{\boldsymbol{k}} c_{\boldsymbol{k} \uparrow}^{\dagger} c_{-\boldsymbol{k} \downarrow}^{\dagger}\right)|0\rangle,
$$

where $|0\rangle$ is the vacuum, and $u_{k}$ and $v_{k}$ are variational parameters, satisfying the condition

$$
\left|u_{k}\right|^{2}+\left|v_{k}\right|^{2}=1 .
$$

$\Pi_{\alpha}$ is a projection operator to partially project out the doubly occupied electron states on each lattice site $i$. The state $\left|\Psi_{\mathrm{GS}}\right\rangle$ may be considered as a generalization of the previously studied partially projected noninteracting electron state $\mathrm{e}^{30-32}$ to include superconductivity. In the limiting case $u_{k} v_{k}=0$, $\left|\Psi_{\mathrm{BCS}}\right\rangle$ is reduced to the noninteracting electron state, and

$$
\left|\Psi_{\mathrm{GS}}\right\rangle \rightarrow \Pi_{\alpha}\left|\Psi_{\mathrm{FL}}\right\rangle,
$$

where $\left|\Psi_{\mathrm{FL}}\right\rangle$ is the ground state of the noninteracting electron system, given by $\left|\Psi_{\mathrm{FL}}\right\rangle=\Pi c_{k \sigma}^{\dagger} c_{k \sigma}|0\rangle$, and the product runs $k, \sigma$

over all the $\boldsymbol{k}$ 's inside the Fermi surface. $\left|\Psi_{\mathrm{GS}}\right\rangle$ is a natural generalization of conventional BCS state to strongly correlated systems. It connects the BCS state to the RVB state, and is characterized by the parameter $\alpha$ between 0 and 1 . $\alpha=0$ corresponds to a conventional BCS state. At $\alpha=1$, the projection operator projects out all the doubly occupied electron states, and $\left|\Psi_{\mathrm{GS}}\right\rangle$ is reduced to the RVB state. ${ }^{3}$ At the half-filling and at $\alpha=1$, each lattice site is occupied by a single electron, and the system is a Mott insulator. Therefore, the wave function $\left|\Psi_{\mathrm{GS}}\right\rangle$ is suitable for studying superconductor-insulator transition. 


\section{THE RENORMALIZED MEAN FIELD THEORY}

We now proceed with the variational calculations to determine the parameters $\alpha$ and $u_{k}, v_{k}$. We consider the electron density $n \leqslant 1$. The variational energy per site $E=\langle H\rangle$ is given by

$$
E=U d+\left\langle H_{t}\right\rangle+\left\langle H_{J}\right\rangle
$$

The first term in Eq. (5) is the intrasite Coulomb interaction energy, while the second and the third terms are the average kinetic and spin-spin correlation energies, respectively. $d$ $=\left\langle n_{i \uparrow} n_{i \downarrow}\right\rangle$ is the average electron double occupation number, and is a function of $\alpha$, and $0 \leqslant d \leqslant 1 / 4$. We have denoted $\langle Q\rangle$ as the expectation value of the operator $Q$ in the state $\left|\Psi_{\mathrm{GS}}\right\rangle$. For briefness, $\left\langle H_{t}\right\rangle,\left\langle H_{J}\right\rangle$ stand for their average values per site.

The variational calculations can be carried out using variational Monte Carlo method. ${ }^{8,33-35}$ Here we use the renormalized Hamiltonian approach to treat the projection operator approximately. ${ }^{7}$ In this approach, the effect of the projection operator is taken into account by a set of classical statistical weighting factors, which multiplies the quantum coherent results of the nonprojected state. This method (Gutzwiller method hereafter) was proposed by Gutzwiller, ${ }^{31}$ and has been applied to study strongly correlated systems by many authors. ${ }^{7,30,32}$ Let $\langle Q\rangle_{0}$ be the expectation value of $Q$ in the state $\left|\Psi_{\mathrm{BCS}}\right\rangle$, then the hopping energy and the spin-spin correlation in the state $\left|\Psi_{\mathrm{GS}}\right\rangle$ are related to those in the state $\left|\Psi_{\mathrm{BCS}}\right\rangle$ by

$$
\begin{aligned}
& \left\langle c_{i \sigma}^{\dagger} c_{j \sigma}\right\rangle=g_{t}\left\langle c_{i \sigma}^{\dagger} c_{j \sigma}\right\rangle_{0}, \\
& \left\langle\boldsymbol{S}_{i} \cdot \boldsymbol{S}_{j}\right\rangle=g_{s}\left\langle\boldsymbol{S}_{i} \cdot \boldsymbol{S}_{j}\right\rangle_{0} .
\end{aligned}
$$

The renormalized factors $g_{t}$ and $g_{s}$ are determined by the ratio of the probability of the physical processes in the states $\left|\Psi_{\mathrm{GS}}\right\rangle$ and $\left|\Psi_{\mathrm{BCS}}\right\rangle$. Following the counting method described in the literature, ${ }^{7}$ we have

$$
\begin{gathered}
g_{t}=\frac{(n-2 d)(\sqrt{d}+\sqrt{1-n+d})^{2}}{(1-n / 2) n}, \\
g_{s}=\frac{(n-2 d)^{2}}{(1-n / 2)^{2} n^{2}} .
\end{gathered}
$$

The expression for $g_{t}$ is the same as in the early literature. ${ }^{30}$ In the limit $d=0$, Eqs. (7) recover the results derived for the $t-J$ model. ${ }^{7}$ These renormalized factors quantitatively describe the correlation effect of the on-site repulsion. $g_{t} \leqslant 1$, and $g_{t} \ll 1$ at small $d$ and small $\delta$, indicating the reduction of the kinetic energy due to the projection. $4 \geqslant g_{s} \geqslant 1$, and $g_{s}$ $=4$ at $d=0$ and $\delta=0$, indicating the enhancement of the spinspin correlation due to the projection. In Fig. 1, we plot $g_{t}$ and $g_{s}$ as functions of the double occupation number $d$ for various electron densities.

In terms of these renormalization factors, we define a renormalized Hamiltonian,

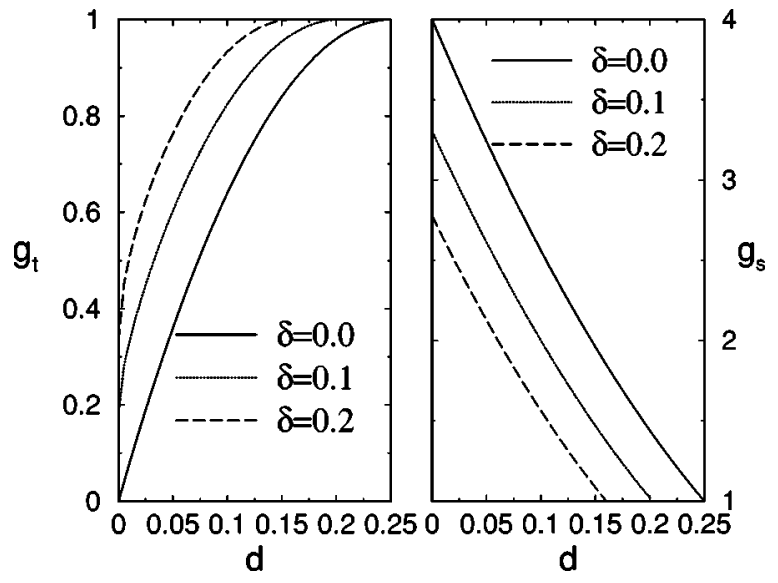

FIG. 1. Gutzwiller's renormalization factors $g_{t}$ and $g_{s}$ as functions of double occupation number $d$ obtained from Eqs. (7). $\delta=1-n$.

$$
H^{\prime}=g_{t} H_{t}+g_{s} H_{s}+H_{U}
$$

The expectation value of $H$ in the state $\left|\Psi_{\mathrm{GS}}\right\rangle$ can thus be evaluated in terms of the expectation value of $H^{\prime}$ in the state $\left|\Psi_{\mathrm{BCS}}\right\rangle$. We obtain,

$$
E=\left\langle H^{\prime}\right\rangle_{0}=U d+g_{t}\left\langle H_{t}\right\rangle_{0}+g_{s}\left\langle H_{J}\right\rangle_{0} .
$$

In the renormalized Hamiltonian approach, the original variational parameters $\left\{\alpha, v_{k}, u_{k}\right\}$ are transformed into the variational parameters $\left\{d, v_{k}, u_{k}\right\}$. There is one-to-one correspondence between $\alpha$ and $d$. Within the Gutzwiller approximation, one can analytically calculate $d=\left\langle n_{i \uparrow} n_{i \downarrow}\right\rangle$, and one finds, ${ }^{31,32}$

$$
(1-\alpha)^{2}=\frac{d(1-n+d)}{(n / 2-d)^{2}} .
$$

This relation is useful to make connections between the Gutzwiller method we adopt here where the partial projection is described by $d$ and Laughlin's method where the partial projection is characterized by $\alpha$.

The formalism below is similar to the renormalized mean field theory developed for the $t-J$ model $^{7}$ except that $d$ may be nonzero in the present theory. We introduce a Lagrangian multiplier $\tilde{\mu}$, and define

$$
K=H^{\prime}-\widetilde{\mu}\left(\sum_{i \sigma} n_{i \sigma}-N_{e}\right),
$$

with $N_{e}$ the number of electrons. We then have $E=\langle K\rangle_{0}$, subject to the condition $\partial\langle K\rangle_{0} / \partial \widetilde{\mu}=0$, or

$$
2 \sum_{k} v_{k}^{2}=n
$$

Below we consider the case $u_{k}$ and $v_{k}$ to be real. Evaluating Eq. (11), we obtain (lattice constant=1), 


$$
\begin{aligned}
E= & U d+\tilde{\mu}+2 \sum_{\boldsymbol{k}}\left(g_{t} \epsilon_{\boldsymbol{k}}-\tilde{\mu}\right) v_{\boldsymbol{k}}^{2} \\
& +\sum_{\boldsymbol{k}, \boldsymbol{k}^{\prime}} V_{\boldsymbol{k}-\boldsymbol{k}^{\prime}}\left(v_{\boldsymbol{k}}^{2} v_{\boldsymbol{k}^{\prime}}^{2}+u_{\boldsymbol{k}} v_{\boldsymbol{k}} u_{\boldsymbol{k}^{\prime}} v_{\boldsymbol{k}^{\prime}}\right),
\end{aligned}
$$

where

$$
\begin{gathered}
V_{\boldsymbol{k}}=-\frac{3}{2} g_{s} J\left(\cos k_{x}+\cos k_{y}\right), \\
\epsilon_{\boldsymbol{k}}=-2 t\left(\cos k_{x}+\cos k_{y}\right) .
\end{gathered}
$$

Carrying out the variational procedure with respect to $u_{k}$ and $v_{k}$, we obtain

$$
\begin{aligned}
& u_{k}^{2}=\frac{1}{2}\left(1+\chi_{k} / E_{k}\right), \\
& v_{k}^{2}=\frac{1}{2}\left(1-\chi_{k} / E_{k}\right),
\end{aligned}
$$

where

$$
E_{k}=\sqrt{\chi_{k}^{2}+\Delta_{k}^{2}}
$$

The variational parameters $\Delta_{k}$ and $\chi_{k}$ are related to the particle-particle pairing amplitude $\Delta_{k}$ and the particle-hole amplitudes $\chi_{k}$ by

$$
\begin{gathered}
\Delta_{k}=\Delta_{x} \cos k_{x}+\Delta_{y} \cos k_{y}, \\
\chi_{k}=\tilde{\epsilon}_{k}-\left(\chi_{x} \cos k_{x}+\chi_{y} \cos k_{y}\right) .
\end{gathered}
$$

In the above equations, we have introduced two correlation functions in the unprojected state $\left|\Psi_{\mathrm{BCS}}\right\rangle$,

$$
\begin{gathered}
\Delta_{\tau}=\left\langle c_{i \downarrow} c_{i+\tau, \uparrow}-c_{i \uparrow} c_{i+\tau, \downarrow}\right\rangle_{0}, \\
\chi_{\tau}=\sum_{\sigma}\left\langle c_{i \sigma}^{\dagger} c_{i+\tau \sigma}\right\rangle_{0},
\end{gathered}
$$

with $\tau=x, y$, the unit vectors on the lattice, and

$$
\tilde{\epsilon}_{k}=\left[-2 g_{t} t\left(\cos k_{x}+\cos k_{y}\right)-\tilde{\mu}\right] /\left(3 g_{s} J / 4\right) .
$$

For the $d$-wave pairing state, which is expected to have the lowest energy within this class of states as suggested in the previous studies for the $t$ - $J$ model, $, 9,9,10,36,37$ we have $\Delta_{x}=$ $-\Delta_{y}=\Delta$, and $\chi_{x}=\chi_{y}=\chi . \Delta$ and $\chi$ are determined by the coupled gap equations,

$$
\begin{gathered}
\Delta=\sum_{\mathbf{k}}\left(\cos k_{x}\right) \Delta_{k} / E_{k}, \\
\chi=-\sum_{\mathbf{k}}\left(\cos k_{x}\right) \chi_{k} / E_{k} .
\end{gathered}
$$

These gap equations must be solved simultaneously with the hole concentration equation, Eq. (12), which can be rewritten as $\delta=\sum \chi_{k} / E_{k}$, with $\delta=1-n$. The variation with respect to k

$d$ leads to the equation

$$
\frac{\partial E}{\partial d}=U+\frac{\partial g_{t}}{\partial d}\left\langle H_{t}\right\rangle_{0}+\frac{\partial g_{s}}{\partial d}\left\langle H_{J}\right\rangle_{0}=0 .
$$

In terms of $\chi$ and $\Delta$, the energy is given by

$$
E=U d-4 n g_{t} t \chi-\left(3 g_{s} J / 4\right)\left(\Delta^{2}+\chi^{2}\right),
$$

where $\chi$ and $\Delta$ are the solutions of the gap equations, and both are functions of $d$. In case Eq. (21) has multiple solutions for $d$, the ground state is determined by the global energy minimum. Alternatively, we may solve the gap equations for given values of $d$, and calculate $E(d)$ to find the optimal value of $d$ to determine the ground state and the ground state energy. The chemical potential of the system, $\mu=\partial E / \partial n$, is given by

$$
\mu=\tilde{\mu}+\frac{\partial g_{t}}{\partial n}\left\langle H_{t}\right\rangle_{0}+\frac{\partial g_{s}}{\partial n}\left\langle H_{J}\right\rangle_{0} .
$$

Note that chemical potential here is different from the Lagrangian multiplier $\tilde{\mu}$ in the renormalized mean field theory, because the renormalized factors $g_{t}, g_{s}$ are also functions of electron density $n$.

\section{MOTT INSULATOR-GOSSAMER SUPERCONDUCTOR TRANSITION}

In this section, we discuss the variational solutions at the half filled case. At the half-filling, the trial wave function $\left|\Psi_{\mathrm{GS}}\right\rangle$ describes either a Mott insulator if $\alpha=1$ (i.e., $d=0$ ), or a SC state if $\alpha<1$ (i.e., $d>0$ ). If $\alpha$ is close to 1 , or $d$ is very close to zero, $\left|\Psi_{\mathrm{GS}}\right\rangle$ describes a Gossamer SC state.

We expect a Mott insulator at large $U$ and a SC state at small $U$. This can be examined qualitatively without carrying out the quantitative calculations. At the half-filling, $g_{t}$ $=8(1-2 d) d$, and $g_{s}=4(1-2 d)^{2}$. Equation (21) becomes

$$
U+8(1-4 d)\left\langle H_{t}\right\rangle_{0}-16(1-2 d)\left\langle H_{J}\right\rangle_{0}=0 .
$$

Since both $\left\langle H_{t}\right\rangle_{0}$ and $\left\langle H_{J}\right\rangle_{0}$ are finite, there will be no solution of Eq. (24) if $U$ is sufficiently large. This indicates that the ground state corresponds to either $d=0$ or $d=d_{\max }$, the allowed maximum value of $d$. The repulsive nature of $U$ excludes the latter, and it follows that the Mott insulating state with $d=0$ is the ground state. We believe that the qualitative result for the existence of the Mott insulating phase at large but finite $U$ is robust. Note that in the Gutzwiller's wave function, the doubly occupied site and the empty site are not correlated. At the half-filling, $d$ represents the carrier density $n^{*}$ and is proportional to the Drude weight in the a.c. conductivity, $n^{*} e^{2} / m^{*}$, with $m^{*}$ the effective mass. We remark that the parameter $d$ in our Gutzwiller approach is different from the usual double occupation number $\tilde{d}$ (for example, the double occupation calculated in the exact diagonalization of a finite size system). In the latter case, $\tilde{d}$ also includes the contribution from the virtual hopping process, hence the double occupied site can be bound to the empty site and the double occupation number $\tilde{d}$ does not represent the mobile carriers.

In the insulating phase $d=0$, so the Hamiltonian is reduced to a Heisenberg model. Within our mean field theory, the RVB ground state energy is given by

$$
E_{0}=-3 J\left(\Delta_{0}^{2}+\chi_{0}^{2}\right)
$$

with 


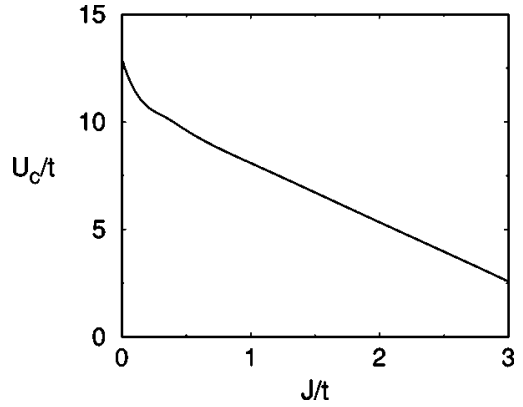

FIG. 2. Phase diagram at half-filling: Insulator at the upper part and superconductor at the lower part.

$$
\Delta_{0}=\chi_{0}=C / \sqrt{2}=\frac{1}{\sqrt{8}} \sum_{k} \sqrt{\cos ^{2} k_{x}+\cos ^{2} k_{y}}=0.339 .
$$

At small $U$, one expects a metallic ground state, except in the special cases due to the band effect such as the von Hove singularity and perfect nesting in $H_{t}$. In this paper, we will not consider the special band effect. We expect a metalinsulator transition at a finite $U=U_{c}$ in the general case, with the metallic phase to be SC provided that $u_{k} v_{k} \neq 0$. This is the Mott insulator-Gossamer superconductor transition.

We now discuss the phase transition in detail. We solve the gap equations for the fixed $d$ and determine the transition point $U_{c}$ and the nature of the transition. The phase diagram in the parameter space $U$ and $J / t$ is plotted in Fig. 2. The critical $U_{c}$ separates the Mott insulating phase from the Gossamer SC phase. We may choose the mobile carrier density as the order parameter, which is proportional to $d$. The phase transition is classified as second type if $d \rightarrow 0$ and first type if $d \rightarrow d_{c}>0$ as $U \rightarrow U_{c}$ from the metallic side. This classification is consistent with the usual zero temperature quantum phase transition, where the nature of the phase transition depends on the continuity or discontinuity of the order parameter. We find the transition to be first order at $0<J / t<\eta_{c}$, and second order at $\eta_{c}<J / t$, with $\eta_{c} \approx 2$. At $J=0$, the present theory is reduced to the Brinkman-Rice theory for metal-insulator transition ${ }^{30}$ for the projected noninteracting electron state. In that case, we find $U_{c} / t=128 / \pi^{2}$. From Fig. 2, we see that $U_{c}(J \rightarrow 0)=U_{c}(J=0)$, so that the critical value of $U$ is continuous at $J=0$. However, the transition is second

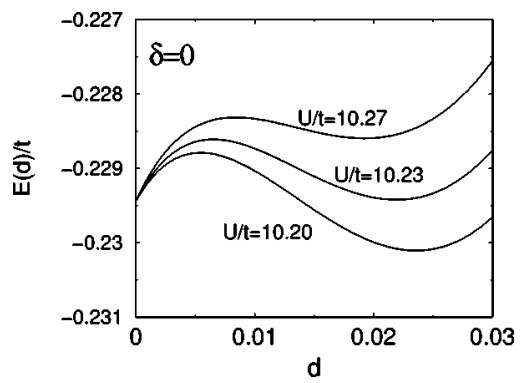

FIG. 3. The energy $E$ as a function of double occupation $d$ for three values of $U$ around $U_{c}$ at $\delta=0$ and $J / t=1 / 3$.

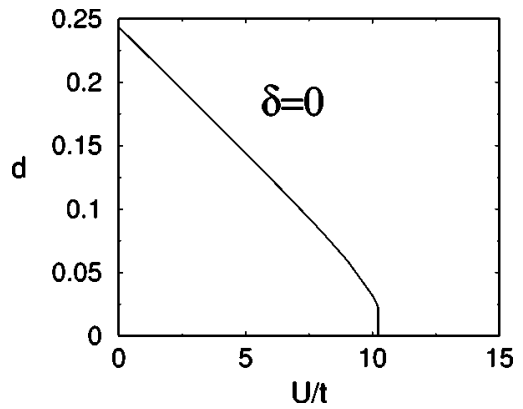

FIG. 4. $d$ as a function of $U$ at $\delta=0$ and $J / t=1 / 3$.

order in the case $J=0$, while it is first order for any small but finite $J / t$.

Let us first discuss the first order phase transition in the region $0<J / t<2$. In Fig. 3, we show the energy $E$ as a function of $d$ at a typical parameter $J / t=1 / 3$. Note that $E$ is not a monotonic function of $d$ around the critical point $U_{c}$. There is a local energy minimum around $d=0.02$, which develops to a global minimum as $U$ approaches $U_{c}$ from the insulator side. The local minimum $E\left(d_{c}\right)$ at $d=d_{c}$ represents a metallic solution, and $E(d=0)$ represents an insulator solution. The critical value for the Mott insulator and Gossamer superconductor transition is determined by the condition $E\left(d_{c}\right)=E(d=0)$. From Fig. 3, we have $U_{c} / t=10.23$ for $J / t$ $=1 / 3$. At $U>U_{c}, d=0$, and the ground state is an insulator. At $U<U_{c}, d \geqslant d_{c} \approx 0.02$, and the ground state is a Gossamer $\mathrm{SC}$ state. As we can see from Fig. 4, $d$ is approximately linear in $U$ except near $U_{c}$, where the discontinuity in $d$ is about 0.02 . We conclude that the Mott insulator-Gossamer superconductor phase transition in this relevant region is first type. The carrier density is discontinuous at the phase transition point. Since $d$ is proportional to the carrier density, this type of first order transition should be observable in the electric transport or in the ac conductivity measurements.

For a large ratio of $J / t$, our calculations show that the phase transition is second order. This is illustrated in Fig. 5 for $E$ vs $d$ in the case of $J / t=3$. The transition occurs at $U_{c}=2.58 t$, and $d$ changes continuously across $U_{c}$.

A special case is $J=0$. In this limit, $\left|\Psi_{\mathrm{GS}}\right\rangle=\Pi_{\alpha}\left|\Psi_{\mathrm{FL}}\right\rangle$, and our theory is reduced to the Brinkman-Rice theory. ${ }^{30}$ The energy at the half-filling is given by

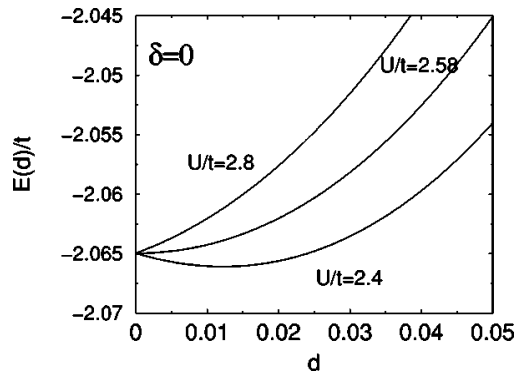

FIG. 5. $E$ as a function of $d$ for several values of $U$ around $U_{c}$ at $\delta=0$ and $J / t=3$. 


$$
E=U d-2 g_{t} \sum_{\mathbf{k}}\left|\cos k_{x}+\cos k_{y}\right|=U d-128 d(1-2 d) t / \pi^{2} .
$$

From this we find $U_{c} / t=128 / \pi^{2} \approx 13 . d$ is continuous at $U_{c}$ so that the transition is a second type.

Our result on the first order phase transition in the physically interesting region (small but nonzero $J / t$ ) is somewhat unexpected. We argue that the first order transition between the Mott insulator and the Gossamer superconductor is due to the interplay of the kinetic and spin-spin correlation energies. This interplay was not included in the previous study of the Gutzwiller approach but is taken into account here. To illustrate this point, we consider the limiting case $0<J / t$ $\ll 1$, and expand the energy $E$ of Eq. (22) at $n=1$ for small $d$,

$$
E(d)=E_{0}+\left(U-U_{c 0}\right) d-\beta d^{2}+O\left(d^{3}\right),
$$

where $E_{0}$ is the energy at $d=0$ given by Eq. (25), $U_{c 0}$ $=16 \sqrt{2} C t-12 C^{2} J$ is the solution of $\partial E / \partial d=0$ at $d=0$, given by Eq. (24). $\beta=\left[32(\partial \chi / \partial d)_{\left.\right|_{d=0}}-32 \sqrt{2} C\right] t$. The $J$ dependence in $\beta$ has been neglected since $J / t \ll 1$. Note that the kinetic energy is proportional to $\chi$. As $d$ increases from $0, \chi$ tends to increase from $\chi_{0}=C / \sqrt{2}$ to gain more kinetic energy. Therefore, $\partial \chi / \partial d>0$. In the limit $J / t \ll 1$, we have $\partial \chi / \partial d=(\chi$ $\left.-\chi_{0}\right) / d \propto t / J \gg 1$, hence the first term in the expression for $\beta$ dominates and $\beta>0$. This demonstrates that $d=0$ is a local maximum in energy at $U=U_{c 0}$, and the phase transition occurs at a large value of $U$ corresponding to $d>0$ as numerically shown in Fig. 3, hence it is a first order transition. Numerically, we find that $\beta=34.8$ in the case $J / t=1 / 3$.

It is interesting to compare the Gossamer superconductorMott insulator transition with the metal-insulator transition studied in previous literature. ${ }^{30}$ In the Brinkman-Rice theory, the transition is second order. In that theory, as the system approaches the insulating phase, the effective mass $m^{*} \rightarrow \infty$. In the Gossamer superconductor-Mott insulator transition with small ratio of $J / t$, the insulating phase is not characterized by the divergence of the effective mass. We estimate the ratio of the effective mass to the band mass $(1 / \mathrm{t})$ at the metallic side of transition point to be $1 / g_{t} \approx 1 /(8 d) \approx 6$.

The first order phase transition between metal and insulator was pointed out by Peierls ${ }^{38}$ and by Landau and Zeldovich ${ }^{39}$ and examined in more great detail by Mott. ${ }^{40} \mathrm{In}$ their theory, an electron is always bound to a positive charge due to the long range Coulomb attraction, and the transition of a metal to an insulator at zero or very low temperatures occurs at a finite critical electron density, and must be the first type. It is interesting to note that the on-site repulsion also leads to the first order transition between a specific type of metal (superconductor) and an insulator studied in the present paper, where the long range Coulomb force is not included. We also note that Florencio and $\mathrm{Chao}^{41}$ investigated the metal-insulator transition of the Hubbard model using Gutzwiller's wave function by including antiferromagnetism and found the transition to be first type.

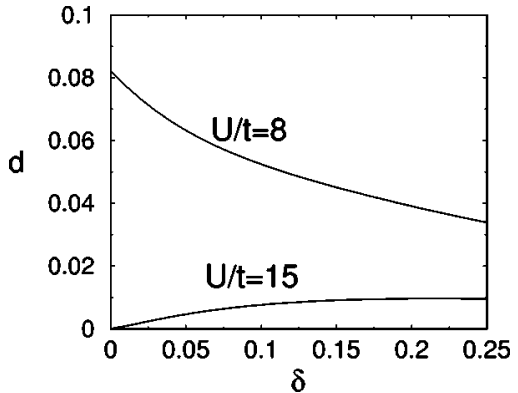

FIG. 6. $d$ as a function of $\delta$ at $J / t=1 / 3$ for $U=8<U_{c}$ and $U$ $=15>U_{c}$.

\section{GOSSAMER AND RVB SUPERCONDUCTIVITY}

In this section, we discuss the SC state at both $\delta=0$ and $\delta>0$. Note that at $\delta>0,\left|\Psi_{\mathrm{GS}}\right\rangle$ always describes a metallic state. To make the terminology clear, we shall call the SC state at $U<U_{c}$ to be a Gossamer superconductor, ${ }^{23,24}$ and the doped Mott insulator ( $U>U_{c}$ and $\delta>0$ ) to be the RVB SC state. $^{3}$

We begin with the discussion of the double occupation number $d$ as a function of the hole concentration. We solve the gap equations and find the optimal value of $d$. The results are plotted in Fig. 6. We find that $d$ is always nonzero at $\delta$ $>0$, even in the region $U>U_{c}$. This suggests that the doped Mott insulator is described by a partially projected state [ $\alpha<1$ in Eq. (2)]. Nevertheless, $d$ is very small for $U$ $\gg U_{c}$. As we can see from Fig. 6, $d$ varies from 0 to 0.01 for $U / t=15$, which corresponds to $U / U_{c} \approx 1.5$. The nonzero value of $d$ at $\delta>0$ may be understood from the variational equation (21), which determines $d$. At $\delta>0, \partial g_{t} / \partial d_{\mid d=0} \rightarrow \infty$. Therefore, $d=0$ cannot be a solution of the equation, and $d$ must be finite. It remains to be seen if this result is due to the Gutzwiller's approximation used in our calculation. It will be interesting to further examine this issue using other methods such as the variational Monte Carlo method.

From Fig. 6, we also see that as $\delta$ increases, $d$ increases for large $U$ but decreases for small $U$. The latter may be understood as follows. In the small $U$ case, the correlation becomes less important, and the qualitative feature between $d$ and $\delta$ becomes similar to the uncorrelated state. For the uncorrelated Fermi liquid state, $d=(1-\delta)^{2} / 4$, so that $d$ monotonically decreases as $\delta$ increases.

While $d$ is a smooth function of $\delta$ for most values of $U$ in our study, there is a narrow region in $U>U_{c}$, where $d$ changes discontinuously at a very small $\delta$. In Fig. 7, we show the energy $E$ vs $d$ for $U=10.235 t$, which is slightly above $U_{c}=10.23 t$, for four values of $\delta$. At $\delta=0$, there is a global energy minimum at $d=0$ and a local minimum around $d=0.02$. As $\delta$ gradually increases, the positions of the two minima change smoothly and their corresponding energies reverse the order. In this region, the optimal value of $d$ jumps. This region is found very narrow: $10.23 t<U$ $<10.235 t$, however.

We now discuss the SC order parameter. The SC order parameter of the state $\left|\Psi_{\mathrm{GS}}\right\rangle$ is defined by, for the $d$-wave pairing, 


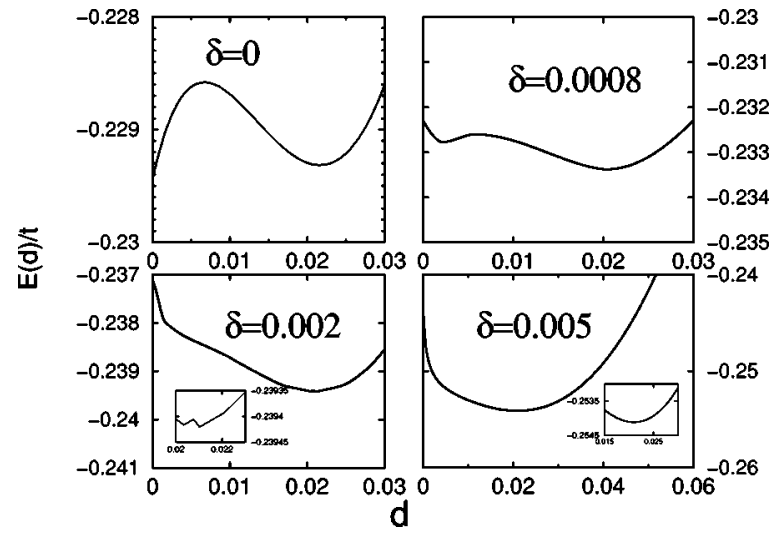

FIG. 7. $E$ as a function of $d$ for $U=10.235 t \geqq U_{c}$ at $J / t=1 / 3$ for several values of $\delta$.

$$
\Delta_{\mathrm{sc}}(\tau)=\left\langle c_{i \downarrow} c_{i+\tau \uparrow}\right\rangle-\left\langle c_{i \uparrow} c_{i+\tau \downarrow}\right\rangle,
$$

and $\Delta_{\mathrm{sc}}=\Delta_{\mathrm{sc}}(x)=-\Delta_{\mathrm{sc}}(y)$. We shall adopt the Gutzwiller approximation to calculate this quantity. In analogy to the derivation for the hopping energy in Eq. (6), we find that ${ }^{7}$

$$
\left\langle c_{i \downarrow} c_{i+\tau \uparrow}\right\rangle=g_{t}\left\langle c_{i \downarrow} c_{i+\tau \uparrow}\right\rangle_{0} .
$$

Therefore, the order parameter $\Delta_{\mathrm{sc}}$ is related to the variational parameter $\Delta$ in the gap equations by

$$
\Delta_{\mathrm{sc}}=g_{t} \Delta
$$

In Fig. 8, we show our results for $\Delta_{\mathrm{sc}}$ and $\Delta$ as functions of $\delta$ for three values of $U$ : well above $U_{c}$, at $U_{c}$, and well below $U_{c}$. Note that at $U=15 t \gg U_{c}, \Delta$ is a maximum but $\Delta_{\mathrm{sc}}=0$ at $\delta=0 . \Delta_{\mathrm{sc}}=0$ is consistent with the Mott insulating ground state. As $\delta$ increases, the kinetic energy plays a more important role, $\Delta$ decreases monotonically. However, $\Delta_{\mathrm{sc}}$ shows a nonmonotonic dome shape for larger $U$. Also note that at $U_{c}$ the Mott insulator and Gossamer SC state are degenerate at $\delta=0$, and the Gossamer SC phase is continuously evolved into the metallic phase at $\delta>0$. Shown in the figure for $U$ $=10.23 t \approx U_{c}$ is the metallic phase. The nonzero value of $\Delta_{\mathrm{sc}}$ at $U=U_{c}$ and $\delta=0$ indicates the transition to be first order.

It is interesting to point out that the SC order parameter

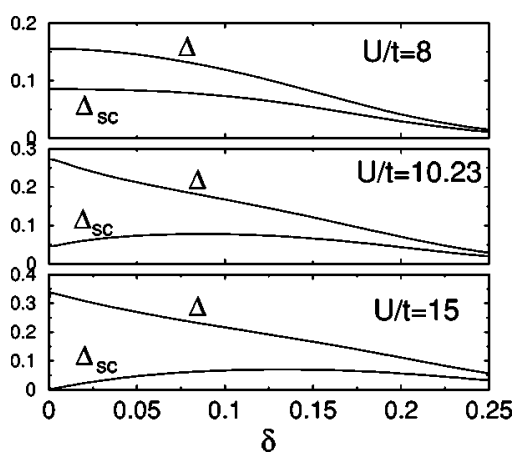

FIG. 8. Variational parameter $\Delta$ and SC order parameter $\Delta_{\mathrm{sc}}$ as functions of $\delta$ for three values of $U$ at $J / t=1 / 3$.

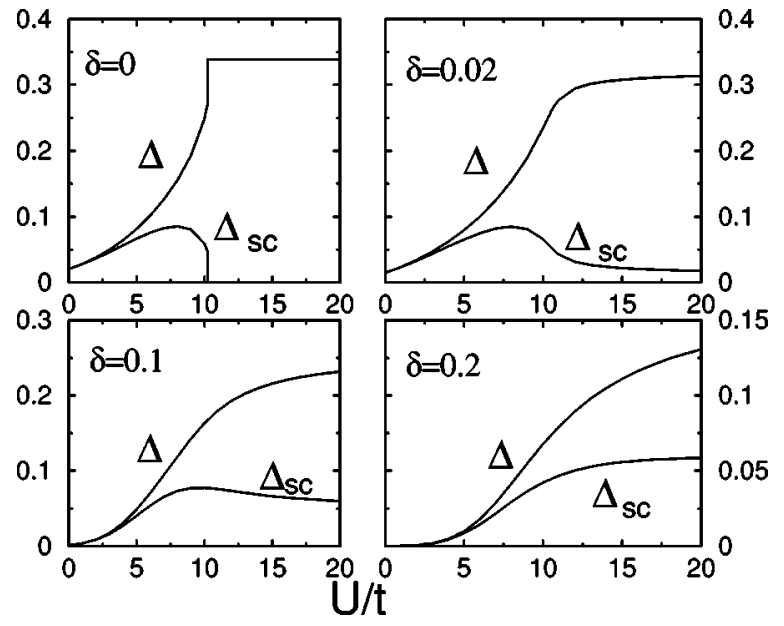

FIG. 9. $\Delta_{\mathrm{sc}}$ and $\Delta$ as functions of $U$ for several values of $\delta$ at $J / t=1 / 3 . \Delta_{\text {sc }}$ changes suddenly at $U_{c}$ in the half-filled case, but changes smoothly as $U$ is reduced from the RVB SC state with $U$ $>U_{c}$ to the Gossamer SC state with $U<U_{c}$. Note that at small values of $U$, the effective Hamiltonian we study here does not represent the original Hubbard model, and nonzero $\Delta_{\mathrm{sc}}$ at $U=0$ is due to the $J$ term in the present model.

$\Delta_{\text {sc }}$ in both the Gossamer and RVB SC states are characterized by the variational parameter $\Delta$ and a small renormalized factor $g_{t}$. They have the same pairing symmetry. It is plausible that the two states are smoothly connected. To examine this issue further, we plot $\Delta_{\mathrm{sc}}$ as a function of $U$ for several values of $\delta$ in Fig. 9. As we can see, at half-filling, $\Delta_{\mathrm{sc}}$ changes suddenly to zero at $U_{c}$, representating a first order phase transition from a Gossamer superconductor to a Mott insulator. However, at $\delta \neq 0, \Delta_{\mathrm{sc}}$ changes continuously across the critical value of $U=U_{c}=10.23 t .{ }^{42}$ This is to say, a RVB SC state at a filling $\delta>0$ is smoothly connected to its corresponding Gossamer SC state at the same $\delta$, and the state with $U>U_{c}$ (the RVB SC) and the state with $U<U_{c}$ (Gossamer $\mathrm{SC})$ are essentially the same at least from the superconductivity point of view. Note that the Gossamer SC state at halffilling and away from the half-filled are smoothly connected, and that the Gossamer SC state is adiabatically connected to the BCS state. ${ }^{23}$ Therefore, our theory suggests that the RVB $\mathrm{SC}$ state is smoothly connected to the Gossamer SC state, hence to the BCS state. This point of view was implied in Ref. 24, and in Ref. 25. The smooth connection between the RVB SC state and the BCS state via Gossamer SC state implies that the RVB state has its genesis in the BCS state, and is in some ways rather conventional. What is unusual is the reduction of the superfluid density and the quasiparticle spectral weight. ${ }^{5}$ In Fig. 10, we present a schematical ground state phase diagram including the Mott insulator, Gossamer and RVB SC states in the parameter space of Coulomb repulsion $U$ and the hole concentration $\delta$.

While the Gossamer and RVB SC states are essentially the same, the chemical potential $\mu$ in the Gossamer SC state is continuous at $\delta=0$ because of the metallic phase, while $\mu$ is discontinuous at $\delta=0$ because the state at $\delta=0$ is an insulator and the state at any small but finite $\delta$ is a metal within the present theory. 


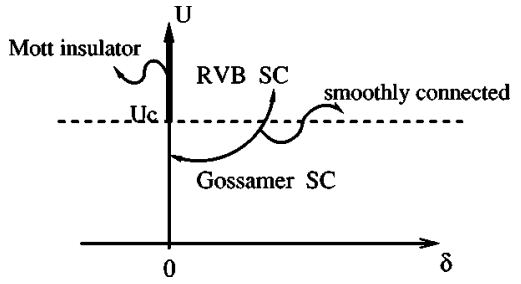

FIG. 10. Schematical phase diagram for the Hamiltonian in parameter space $U$ and $\delta$. The line of $\delta=0$ and $U>U_{c}$ corresponds to the Mott insulating phase. The RVB SC phase is in the region $U$ $>U_{c}$ and $\delta>0$, and the Gossamer SC phase is in the region $U$ $<U_{c}$ with either $\delta=0$ or $\delta>0$.

Below we shall study $\mu$ quantitatively. At $\delta=0, \mu=U / 2$ by electron-hole symmetry. At other values of $\delta$, we calculate $\mu$ using Eq. (23) after solving the gap equations. In Fig. 11, we show $\mu$ as a function of $\delta$. As we can see from the figure, $\mu=U / 2$ at $\delta=0$, and is continuous for $U \leqslant U_{c}=10.23 t$. There is a discontinuity in $\mu$ for $U>U_{c}$ at $\delta=0$. At $U>U_{c}$, the chemical potential is shifted from $U / 2$ at the half-filled to the lower Hubbard band away from the half-filled. To see this more explicitly, we define $\Delta \mu=\mu(d \rightarrow 0)-\mu(d=0) . \Delta \mu$ as a function of $U$ is plotted in Fig. 12. As $U$ decreases, $\Delta \mu$ decreases monotonically and reaches a finite value at $U$ $=U_{c}+0^{+}$, then drops to zero at $U=U_{c}-0^{+}$. The discontinuity of $\Delta \mu$ at $U_{c}$ is related to the first order phase transition.

Finally, we briefly discuss the excited states. In the context of the Gutzwiller trial wave function, the excited states were discussed by Zhang et $a .^{7}$ for the RVB state, and recently by Laughlin for a Gossamer SC Hamiltonian. ${ }^{23}$ Here we follow Ref. 7 to discuss quasiparticle states in the Gossamer superconductor. We consider quasiparticle state,

$$
\left|\Psi_{\boldsymbol{p} \uparrow}\right\rangle=\prod_{\alpha} c_{\boldsymbol{p} \uparrow}^{\dagger} \prod_{\boldsymbol{k} \neq \boldsymbol{p}}\left(u_{\boldsymbol{k}}+v_{\boldsymbol{k}} c_{\boldsymbol{k} \uparrow}^{\dagger} c_{-\boldsymbol{k} \downarrow}^{\dagger}\right)|0\rangle
$$

The quasiparticle energy $\widetilde{E}_{p}$ is defined to be the difference of the expectation values of $K$ [see Eq. (11) ] in this state and in the ground state $\left|\Psi_{\mathrm{GS}}\right\rangle$. We use the Gutzwiller method to calculate the energy and obtain ${ }^{7}$

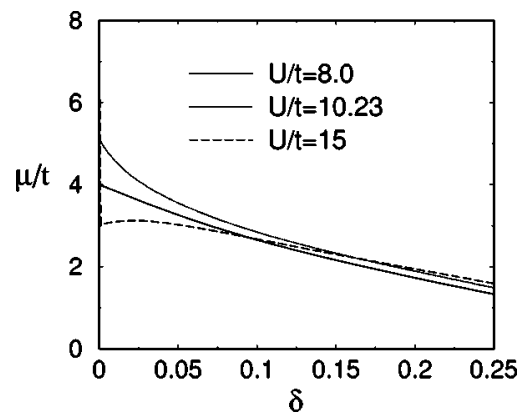

FIG. 11. The chemical potential $\mu$ as a function of $\delta$ for three values of $U$ at $J / t=1 / 3$.

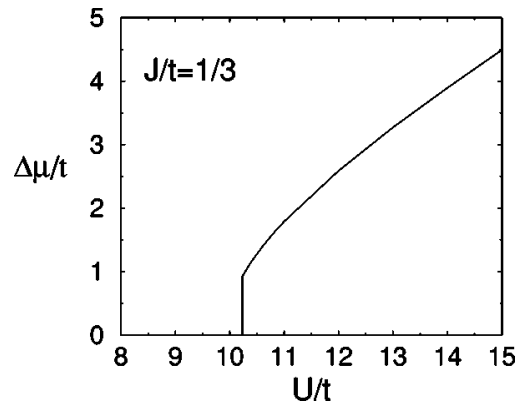

FIG. 12. The discontinuous in chemical potential as a function of $U$.

$$
\widetilde{E}_{\boldsymbol{p}}=\left(3 g_{s} J / 4\right) \sqrt{\chi_{\boldsymbol{p}}^{2}+\Delta_{p}^{2}}
$$

At the wave vector $\boldsymbol{p}$ satisfying $\chi_{p}=0$ [Eq. (17)], we have $\widetilde{E}_{p}=\Delta\left|\cos k_{x}-\cos k_{y}\right|$. Therefore, the quasiparticle energy is proportional to the parameter $\Delta$, which is not renormalized by the factor $g_{t}$, in sharp contrast to the SC order parameter which is renormalized down by a factor of $g_{t}$. Our result here is consistent with Refs. 7 and 23. Since $E_{p}$ is not renormalized, we can see from Fig. 8 that the quasiparticle energy is maximum at $\delta=0$, and decreases as doping concentration increases. This feature was first found for the $t-j$ model, ${ }^{7}$ and is consistent with the "high energy pseudogap" observed in the angular resolved photoemission experiments and the observed SC energy gap. ${ }^{43-45}$ Here we show that this feature should also appear in the Gossamer superconductor.

\section{SUMMARY}

We have used the Gutzwiller variational method to study an effective Hamiltonian for the Hubbard model in a square lattice. Based on the Gutzwiller approximation, we have discussed the ground state both at half-filling and away from the half-filled. At the half-filling, there is a first order phase transition to separate a Mott insulator at large Coulomb repulsion $U$ from a Gossamer superconductor at small $U$. This is very interesting. It suggests that the on-site Coulomb repulsion can lead to a first order transition between a specific type of metal and an insulator. The double occupation number $d$, which is proportional to the carrier density, changes discontinuously from zero in the Mott insulator phase to a finite value in the metallic phase at the phase transition point $(U$ $\left.=U_{c}\right)$. We expect that this type of first order transition should be observable in the electric transport or in the ac conductivity measurements. Away from the half-filled, the Gutzwiller variational state is always metallic. The Gossamer SC state changes continuously, while the Mott insulating phase becomes RVB SC. The Gossamer superconductor is similar to the RVB SC state with the same type of pairing symmetry and similar type of pseudogap. They are smoothly connected. Their major difference is on the position of the chemical potentials. The Gutzwiller method we used in this paper has previously been tested in good agreement with the variational Monte Carlo method. ${ }^{7,8}$ We believe that the qualitative 
conclusions obtained here should be reliable, and refined numerical calculations such as variational Monte Carlo calculations will be interesting for further examination of the problem. There are other questions that require further investigation, such as the competition with the antiferromagnetic phase, which will be for our future study.

\section{ACKNOWLEDGMENTS}

We wish to thank R. Laughlin, T. M. Rice, P. W. Anderson, Y. Yamashita, and Y. Yu for many interesting discussions. This work was partially supported by the U.S. NSF Grant No. 0113574, by the Chinese Academy of Sciences, and by RGC in Hong Kong.
${ }^{1}$ J. G. Bednorz and K. A. Muller, Z. Phys. B: Condens. Matter 64, 189 (1986)

${ }^{2}$ M. K. Wu et al., Phys. Rev. Lett. 58, 908 (1987).

${ }^{3}$ P. W. Anderson, Science 235, 1196 (1987).

${ }^{4}$ F. C. Zhang and T. M. Rice, Phys. Rev. B 37, 3759 (1988).

${ }^{5}$ P. W. Anderson, P. A. Lee, M. Randeria, T. M. Rice, N. Trievedi, and F. C. Zhang, J. Phys.: Condens. Matter 24, R755 (2004).

${ }^{6}$ G. Baskaran, Z. Zou, and P. W. Anderson, Solid State Commun. 63, 973 (1987).

${ }^{7}$ F. C. Zhang, C. Gros, T. M. Rice, and H. Shiba, Supercond. Sci. Technol. 1, 36 (1988).

${ }^{8}$ C. Gros, Phys. Rev. B 38, 931 (1988); H. Yokoyama and H. Shiba, J. Phys. Soc. Jpn. 57, 2482 (1988); C. Gros, Ann. Phys. (N.Y.) 189, 53 (1989).

${ }^{9}$ G. Kotliar and J. Liu, Phys. Rev. B 38, 5142 (1988).

${ }^{10}$ H. Fukuyama, Prog. Theor. Phys. Suppl. 108, 287 (1992).

${ }^{11}$ P. A. Lee, Physica C 317, 194 (1999); N. Nogaosa and P. A. Lee, Phys. Rev. Lett. 64, 2450 (1990); X. G. Wen and P. A. Lee, ibid. 76, 503 (1996).

${ }^{12}$ A. Paramekanti, M. Randeria, and N. Trivedi, Phys. Rev. Lett. 87, 217002 (2001); A. Paramekanti et al., cond-mat/0305611 (unpublished)

${ }^{13}$ D. J. Van Harlingen, Rev. Mod. Phys. 67, 515 (1995).

${ }^{14}$ C. C. Tsuei and J. R. Kirtley, Rev. Mod. Phys. 72, 969 (2000).

${ }^{15}$ A. Damascelli, Z. Hussain, and Z. X. Shen, Rev. Mod. Phys. 75, 473 (2003); T. Timusk and B. Statt, Rep. Prog. Phys. 62, 61 (1999).

${ }^{16}$ Y. J. Uemura, Phys. Rev. Lett. 62, 2317 (1989).

${ }^{17}$ S. Sorella, G. B. Martins, F. Becca, C. Gazza, L. Capriotti, A. Parola, and E. Dagotto, Phys. Rev. Lett. 88, 117002 (2002).

${ }^{18}$ C. T. Shih, Y. C. Chen, H. Q. Lin, and T. K. Lee, Phys. Rev. Lett. 81, 1294 (1998).

${ }^{19}$ F. F. Assaad, W. Hanke, and D. J. Scalapino, Phys. Rev. B 50, 12835 (1994).

${ }^{20}$ S. Zhang, J. Carlson, and J. E. Gubernatis, Phys. Rev. Lett. 78, 4486 (1997).

${ }^{21}$ S. R. White and D. J. Scalapino, Phys. Rev. B 60, R753 (1999).

${ }^{22}$ Th. Maier, M. Jarrell, Th. Pruschke, and J. Keller, Phys. Rev. Lett. 85, 1524 (2000); M. Jarrell, Th. Maier, M. H. Hettler, and
A. N. Tahvildarzadeh, Europhys. Lett. 56, 563 (2001).

${ }^{23}$ R. Laughlin, LANL cond-mat/0209269 (unpublished).

${ }^{24}$ F. C. Zhang, Phys. Rev. Lett. 90, 207002 (2003).

${ }^{25}$ G. Baskaran, Phys. Rev. Lett. 90, 197007 (2003).

${ }^{26}$ B. A. Bernevig, R. B. Laughlin, and D. I. Santiago, Phys. Rev. Lett. 91, 147003 (2003); B. A. Bernevig, G. Chapline, R. Laughlin, Z. Nazario, and D. Santiago, cond-mat/0312573 (unpublished).

${ }^{27}$ See also Ref. 25 . Note that the numbers of empty and doubly occupied sites in the present theory are fixed only in average, while they are rigorously fixed in Ref. 25.

${ }^{28}$ D. Jerome, Science 252, 1509 (1991); S. Lefebvre, P. Wzietek, S. Brown, C. Bourbonnais, D. Jrome, C. Mzire, M. Fourmigu, and P. Batail, Phys. Rev. Lett. 85, 5420 (2000).

${ }^{29} \mathrm{C}$. Honerkamp (private communication).

${ }^{30}$ W. Brinkman and T. M. Rice, Phys. Rev. B 2, 4302 (1970).

${ }^{31}$ M. C. Gutzwiller, Phys. Rev. 137, A1726 (1965).

${ }^{32}$ D. Vollhardt, Rev. Mod. Phys. 56, 99 (1984).

${ }^{33}$ C. Gros, R. Joynt, and T. M. Rice, Phys. Rev. B 36, 381 (1987); G. J. Chen, R. Joynt, F. C. Zhang, and C. Gros, ibid. 42, 2662 (1990).

${ }^{34}$ H. Yokoyama and H. Shiba, J. Phys. Soc. Jpn. 56, 3570 (1987).

${ }^{35}$ A. Himeda and M. Ogata, Phys. Rev. B 60, R9935 (1999).

${ }^{36}$ I. Affleck, Z. Zou, T. Hsu, and P. W. Anderson, Phys. Rev. B 38, 745 (1988)

${ }^{37}$ F. C. Zhang, Phys. Rev. Lett. 64, 974 (1990).

${ }^{38} \mathrm{R}$. Peierls, see the quote in the reference of Landau and Zeldovich below.

${ }^{39}$ L. Landau and G. Zeldovich, Acta Physicochim. URSS 18, 194 (1943).

${ }^{40}$ N. F. Mott, in Metal-Insulator Transition, 2nd ed. (Taylor and Francis, New York, 1990), p. 127.

${ }^{41}$ J. Florencio and K. A. Chao, Phys. Rev. B 14, 3121 (1976).

${ }^{42}$ At very small $\delta<0.005$, numerical calculations show somewhat dramatic change in $\Delta_{\mathrm{sc}}$ at a value of $U$ nearby $U_{c}$, similar to what is discussed in Fig. 7.

${ }^{43}$ M. R. Norman and M. Randeria, Phys. Rev. B 52, 615 (1995).

${ }^{44}$ Z. X. Shen et al., Phys. Rev. Lett. 70, 1553 (1993).

${ }^{45}$ J. C. Campuzano et al., Phys. Rev. Lett. 83, 3709 (1999). 